\title{
Experimental and numerical approach to identify major heating mechanism in induction thermography of carbon fiber reinforced plastic components
}

\author{
by Renil Thomas K*, C V Krishnamurthy**, Krishnan Balasubramanian*
}

\author{
* Department of Mechanical Engineering, Indian Institute of Technology Madras, Chennai, 600036, India, \\ ktrenil@gmail.com \\ ${ }^{* \star}$ Department of Physics, Indian Institute of Technology Madras, Chennai, 600036, India.
}

\begin{abstract}
Induction based heating is a promising technique for the detection of cracks in electrically conducting structures using thermography. This technique can be extended to composite structures especially carbon fiber reinforced plastic (CFRP) components due to the presence of conducting carbon fibers. However, there have been various conflicting mechanisms suggested in the literature. This paper discusses about the experimental and various numerical approaches to understand the major heating mechanism happening inside the composites
\end{abstract}

\section{Introduction}

Composites are widely used in aerospace, automobile industries due to its low weight and high strength. Nondestructive evaluation of composites is required for assuring the quality of structures. Thermography is a promising technique to detect the common defects like fiber breakage, delamination and matrix crack. Induction based heating is an advanced technique for active thermography for the detection of cracks in electrically conducting structures. This technique can be extended to composite structures especially carbon fiber reinforced plastic (CFRP) components due to the presence of conducting carbon fibers. However, there have been various conflicting mechanisms suggested in the literature.

The initial works explaining heating mechanisms on composites were reported by Miller et al. [1]-[3] They used this technique for the die-less fabrication of composites which requires rapid and localized heat generation. According to them, the dominant heating mechanism was joule losses in the carbon fibres when the current pass through the conductive loops. Later, Fink et al. proposed a new theory of dielectric losses in the polymer at the junctions of fiber layers [4]. They developed various models based on this mechanism [5], [6]. After few years Kim et al. and Yarlagadda et al.came up with a more detailed study on various heating mechanisms that can occur on composites [7]-[9]. Thus, three heating mechanisms were discussed in their papers-(1) Joule losses in the fibers, (2) Dielectric losses in the polymer, (3) Contact resistance losses at the junctions. composite.

This work proposes an experimental and numerical approach to identify the major heating mechanism in the

\section{Experiment}

In order to understand the heating behaviour, strips of carbon fibres were considered for the experiment. Width of each strip is $3 \mathrm{~mm}$ and the thickness of the strip is approximately $0.24 \mathrm{~mm}$. Four strips of carbon fibres were arranged in two mutually perpendicular layers to form a 'square loop' configuration of dimension $4 \times 4 \mathrm{~cm}$. Fibres are in physical contact at the intersection regions. Two cases were considered for the study-(1) Closed square loop (figure 1) (2) Open square loop (figure 2). An induction heating unit of $1 \mathrm{~kW}$ output power was used to excite the coil. The coil was excited at $225 \mathrm{kHz}$ frequency with $200 \mathrm{~A}$ current for $1 \mathrm{~s}$. The size of the coil is smaller than the square loop fabricated. A medium wave infrared camera (MWIR) with $320 \times 256$-pixel resolution was used to acquire the temperature information at a frame rate of $100 \mathrm{~Hz}$.

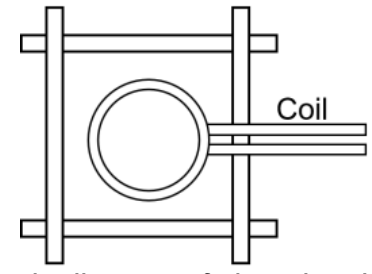

Fig. 1. Schematic diagram of closed carbon fiber loop

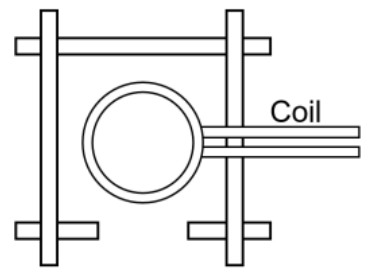

Fig. 2. Schematic diagram of open carbon fiber loop 


\section{Numerical Modelling}

A finite element based (FEM) numerical model was developed to simulate the experimental study using a commercially available FEM package. Multi-physics simulation was performed to calculate the eddy current produced and temperature developed due to the flow of eddy currents. Dimensions of the model were kept same as that of the experiment. Individual fibres in the strip were not modelled as it demands heavy computational resources instead, the strips were modelled as a homogeneous material. In experiment, even though the fibres are in physical contact, there will be a contact resistance existing between the layers. In order to numerically handle this, a different material with less electrical conductivity than the conductive strip was introduced between fiber layers at the junctions

\section{Results and discussion}

Out of two cases considered in the experiment, the first case where a closed loop was existing, the heating was observed at the junctions (figure 3). In the second case where the loop is open, heating cannot be observed anywhere in the configuration. This experiment underlies the fact that a closed loop of fibers with electrical connection at the junctions is necessary for the production of heat. It also attributes to the fact that the predominant heating is happening at the junctions instead of fibers. The developed numerical model also shows junction heating experienced due to the contact resistance.

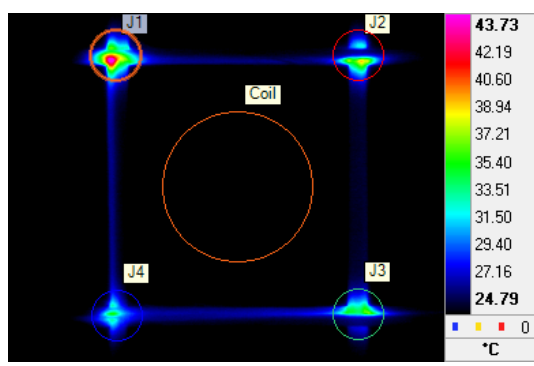

Fig. 3. Thermogram of junction heating phenomenon for a closed loop case

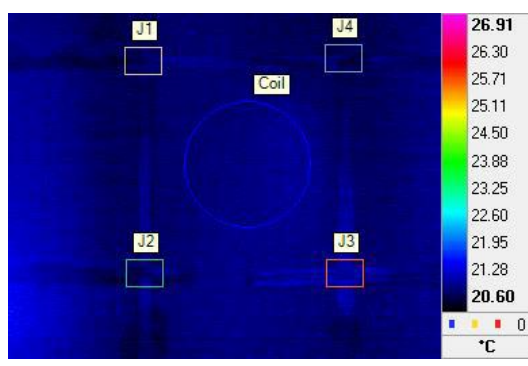

Fig. 4. Thermogram of an open loop case

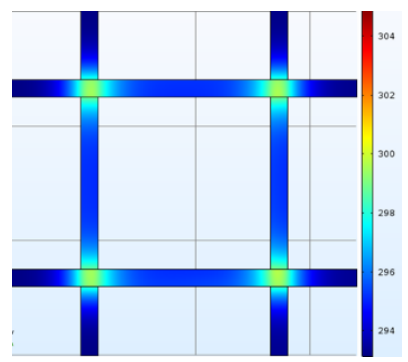

Fig. 5. Numerical result showing junction heating phenomenon

\section{Conclusion}

The experiment proposed in this paper shows that the major heating mechanism occurring in the composite is junction heating due to contact resistance. The existence of a global current flow path is necessary for the flow of eddy currents in the fibers which leads to heating phenomenon. This observation can be used to visualise various defects formed in the composite especially fiber cracks. Those defect zones may offer contact resistance for the flow of eddy current which eventually results in the generation of localised heat which helps thermography technique to image the defects. The proposed numerical model shows close resemblance with the experimental observations. Thus, the physical phenomenon of the contact resistance at the junctions can be numerically handled by introducing a different material with less electrical conductivity between the fibers. This approach cab be extended to model defects in the composite which can be further explored by altering various characteristics of defects such as size, shape, defect type etc.

\section{REFERENCES}

[1] A. K. Miller, C. Chang, A. PAYNE, M. GUR, and E. MENZEL, "The nature of induction heating in graphite-fiber, polymer-matrix composite materials,” SAMPE J., vol. 26, pp. 37-54, 1990.

[2] A. K. Miller, M. Gur, A. Peled, A. Payne, and E. Menzel, "Die-Less forming of thermoplastic matrix continuous fiber composites," Sampe J., vol. 26, no. 4, pp. 37-54, 1990.

[3] W. Lin, O. Buneman, and A. K. Miller, "Induction heating model for graphite fiber/thermoplastic matrix composites," Sampe J., vol. 27, no. 6, pp. 45-51, 1991.

[4] B. K. Fink, R. L. McCullough, and J. W. Gillespie, "A local theory of heating in cross-ply carbon fiber thermoplastic composites by magnetic induction," Polym. Eng. Sci., vol. 32, no. 5, pp. 357-369, 2004.

[5] B. K. Fink, R. L. McCullough, and J. W. Gillespie, "A model to predict the planar electrical potential distribution in cross-ply carbon-fiber composites subjected to alternating magnetic fields," Compos. Sci. Technol., vol. 49, no. 1, pp. 71-80, Jan. 1993.

[6] B. K. Fink, R. L. McCullough, and J. W. Gillespie, "A model to predict the through-thickness distribution of heat generation in cross-ply carbon-fiber composites subjected to alternating magnetic fields," Compos. Sci. Technol., vol. 55, no. 2, pp. 119-130, 1995. 
[7] H. Kim, S. Yarlagadda, J. W. Gillespie, N. B. Shevchenko, and B. K. Fink, "A study on the induction heating of carbon fiber reinforced thermoplastic composites," Adv. Compos. Mater., vol. 11, no. 1, pp. 71-80, 2002.

[8] S. Yarlagadda, H. J. Kim, J. W. Gillespie Jr, N. B. Shevchenko, and B. K. Fink, "A study on the induction heating of conductive fiber reinforced composites," J. Compos. Mater., vol. 36, no. 4, pp. 401-421, 2002.

[9] F. P. Stacks, H. Im, and S. Yarlagadda, "Development of a Numerical Model to Predict In-plane Heat Generation Patterns During Induction Processing of Carbon,” vol. 37, no. 16, 2003. 